\title{
IntroComp: Atraindo Alunos do Ensino Médio para uma Instigante Experiência com a Programação
}

\author{
Leonardo F. Meneses ${ }^{1}$, Luiz Felipe F. Mai ${ }^{1}$, Jordão Rosario ${ }^{1}$, Elias de Oliveira ${ }^{2}$, \\ Roberta L. Gomes ${ }^{1,2}$ \\ ${ }^{1}$ Departamento de Informática , PET Engenharia de Computação \\ ${ }^{2}$ Programa de Pós-Graduação em Informática \\ Universidade Federal do Espírito Santo (UFES) \\ Av. Fernando Ferrari, 514, Goiabeiras - 29075-910 - Vitória - ES - Brasil \\ \{leoferreirafx, lffmai, jordao.rosario01\} @gmail.com, \\ eliasdlcad.inf.ufes.br, rgomesdinf.ufes.br
}

\begin{abstract}
Resumo. Com o aumento na demanda por profissionais da área de Computação, e a constatação de um crescente desinteresse por parte dos jovens por cursos ligados ao setor de Tecnologia da Informação, o Brasil caminha para um cenário em que a falta de profissionais de TI poderá causar prejuízos da ordem de bilhões de Reais. Visando justamente atrair mais jovens para a área de Computação, os alunos do PET Engenharia de Computação da UFES criaram o Projeto IntroComp. Esse Projeto promove anualmente para alunos do ensino médio da rede pública da Grande Vitória um curso de Introdução à Programação, seguido de um preparatório para Olimpíada Brasileira de Informática. Este trabalho apresenta um relato de experiência descrevendo as iniciativas e estratégias do Projeto, assim como alguns resultados obtidos em seus cinco anos de existência.
\end{abstract}

\section{Introdução}

Uma consequência direta da introdução da Computação em praticamente todos os setores da Sociedade é a crescente demanda por profissionais dessa área. Isso vem sendo evidenciado por diferentes estudos e estimativas. Por exemplo, de acordo com a agência americana de estatísticas Bureau of Labor Statistics ${ }^{1}$, entre 2010 e 2020, a previsão de aumento na taxa de novos empregos nos Estados Unidos no grupo "Matemática e Computação" é de $22 \%$, maior do que a média dos grupos $(14.3 \%)$ [Lockard 2012].

No caso do Brasil, o aumento na demanda por profissionais de Computação reforça a necessidade de atrair, em grande número, mais interessados para a disciplina. No entanto, observamos um crescente desinteresse por cursos relacionados ao setor de Tecnologia da Informação. Paralelo a isso, os índices de evasão de alunos nos cursos dessa área estão entre os mais altos do País [Schetinger 2009] [Araújo 2013], o que vem acarretando diretamente na escassez de mão de obra disponível no mercado. O governo

\footnotetext{
${ }^{1}$ http://www.bls.gov/
} 
Brasileiro já reconhece que o Brasil pode perder R $\$ 115$ bilhões por ano a partir de 2017 por falta de profissionais de Tecnologia da Informação [SOFTEX 2012].

Além do problema da evasão, observa-se em alguns cursos altos índices de reprovação, principalmente em disciplinas que envolvem programação [Marques 2011]. Esse fato é crítico uma vez que o domínio da programação é uma característica bastante desejável e valorizada nos profissionais. Efetivamente, a programação não é um assunto trivial. Para ser um bom programador, é necessário um conjunto de habilidades técnicas e cognitivas, como bom raciocínio lógico e capacidade de interpretação, além do conhecimento das linguagens de programação em si [Scaico et al. 2012].

Visto que o desenvolvimento de algoritmos e programas exige competências avançadas, quanto mais cedo os alunos tiverem a oportunidade de desenvolver essas habilidades, menor a dificuldade para que as mesmas sejam desenvolvidas. Com isso, trazer o ensino da programação para o ensino médio pode ser uma estratégia importante para se quebrar essa barreira de aprendizado, além de permitir maior fomento de interesse pela Computação, trazendo mais alunos e, consequentemente, mais profissionais para área no país [Pereira Junior et al. 2005].

Nesse contexto, um grupo de alunos do Programa de Educação Tutorial (PET) do curso de Engenharia de Computação da Universidade Federal do Espírito Santo, decidiu iniciar um projeto de ensino e extensão voltado para alunos de nível médio da rede pública (Estadual e Federal), da Grande Vitória. O projeto denominado IntroComp - Introdução à Computação - tem como objetivo geral atrair alunos desse nível para uma instigante experiência com a programação. O curso visa principalmente despertar nos alunos o interesse pela Computação por meio do desenvolvimento do pensamento computacional, sendo este último uma ferramenta importante que pode ser aplicada para resolver problemas nos mais diversos campos do conhecimento, não apenas dentro da Ciência da Computação [Gomes 2013].

O presente trabalho apresenta um relato de experiência descrevendo as iniciativas e estratégias do projeto IntroComp, bem como alguns resultados obtidos em seus cinco anos. Este artigo encontra-se estruturado conforme segue. Na seção 2, é apresentado o projeto IntroComp, incluindo seu histórico e a metodologia aplicada. $\mathrm{Na}$ seção 3 são apresentados os principais resultados do projeto. Na seção 4 são descritas as recentes ações visando a ampliação do IntroComp. Na seção 5 são listados os trabalhos relacionados. Na seção 6 são colocadas algumas conclusões e perspectivas do projeto.

\section{Projeto IntroComp}

O projeto IntroComp - Introdução à Computação, aos moldes de uma estratégia já adotada no Estados Unidos ${ }^{2}$, vem com a premissa principal de levar o ensino de programação a alunos do ensino médio. O foco do IntroComp está nos alunos da rede pública, visto que, geralmente, os programas desenvolvidos pelo Governo Federal visando inclusão digital se limitam ao treinamento no uso de tecnologias (informática básica), e não à programação em si [Garcia 2008].

Promovido e criado no final de 2010 pelo PET Engenharia de Computação da UFES, o IntroComp proporciona aos estudantes a oportunidade de explorar mais a

\footnotetext{
${ }^{2}$ https://code.org/
} 
fundo tecnologias da informação, bem como serem capazes de resolver problemas por meio da computação, instigando nestes características como a criatividade e, principalmente, o raciocínio lógico.

Em sua primeira edição, no ano de 2011, o projeto teve seu primeiro vínculo com uma escola da rede pública de ensino médio. Em 2012, estendeu-se para 4 escolas Estaduais. Em 2013, já abrangia praticamente todas as escolas da rede pública de Vitória - ES, incluindo as instituições federais. Em sua mais recente edição, em 2014, manteve o público atendido. O gráfico ilustrado na Figura 1 apresenta a evolução do número de participantes do projeto em cada uma de suas edições.

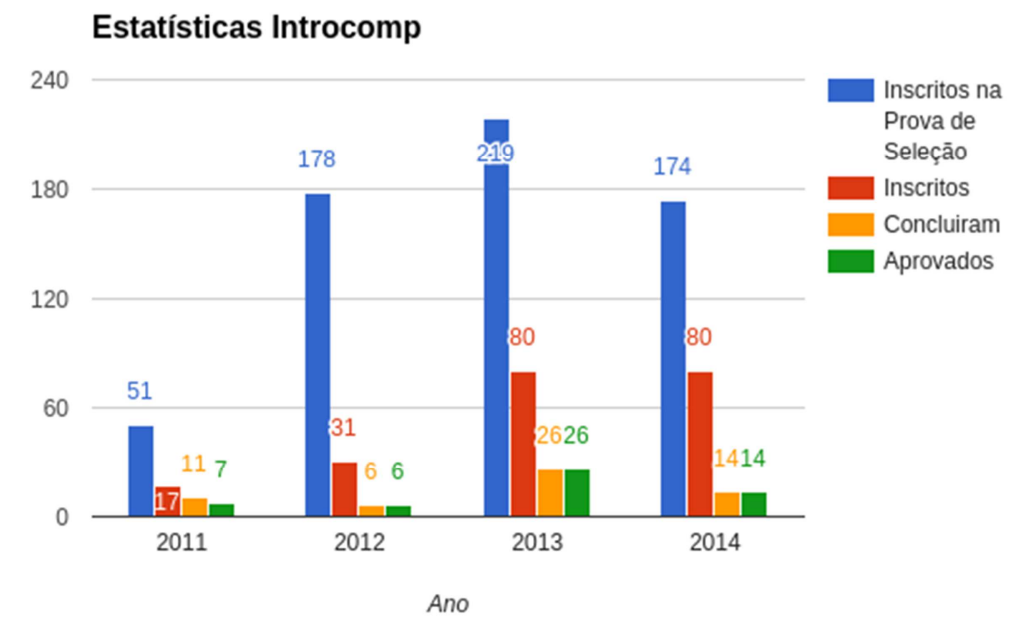

Figura 1: Participação dos alunos entre os anos de 2011 e 2014

\subsection{Metodologia}

No contexto do projeto, é oferecido anualmente o curso de Introdução à Programação, sempre no segundo semestre, com aulas na própria Universidade. A primeira etapa do IntroComp consiste no planejamento do curso e, logo mais, a divulgação. Dentro do planejamento, organiza-se os planos de divulgação, calendário de aulas, e, principalmente, o programa (conteúdo) das aulas. A ordem e o conteúdo das aulas foi escolhida com base no livro "Introdução à Programação: Uma Nova Abordagem Usando C" [Varejão 2014], cuja elaboração e edição contou com a participação de vários alunos envolvidos no projeto IntroComp.

Em junho/julho, os membros do projeto se dividem e comparecem às escolasalvo para divulgar o IntroComp, pregando cartazes pelas escolas e explicando o curso sala a sala (o que é, período de inscrições, processo de seleção, etc.). No ano de 2014, o projeto foi divulgado em 7 escolas: sendo 5 delas Estaduais e 2 delas Federais.

Feito isso, passamos para a etapa de seleção, em que selecionamos 80 alunos dentre todos os inscritos para poderem participar das aulas do IntroComp. A seleção é realizada por meio de uma prova de lógica (nenhum conhecimento prévio é requisitado) com cerca de 25 questões de níveis fácil, mediano e difícil.

$\mathrm{Na}$ primeira semana de agosto iniciam-se as aulas, que ocorrem das $9 \mathrm{~h}$ às $12 \mathrm{~h}$ todos os sábados até o final do curso, por volta do meio de dezembro. No primeiro dia, apresentamos melhor o curso e sua estrutura, a ementa, como serão ministradas as aulas, os professores etc. A partir da segunda semana, os alunos têm seu primeiro contato com a programação e assim prosseguem até o final do ano. 
Ao final de cada aula, disponibilizamos em nosso site todas as aulas, juntamente com os Workings (conjunto de exercícios de fixação), que devem ser feitos em casa e que contam nota para a conclusão do curso. Esses exercícios são submetidos utilizando o Moodle ${ }^{3}$ - um ambiente virtual de aprendizagem - onde, além disso, os alunos encontram espaço para discutir dúvidas, obter materiais extras, além de poder enviar mensagens diretamente aos professores. A escolha do Moodle se deu principalmente por ser um software gratuito, leve e eficiente, nos permitindo agrupar as atividades avaliativas em categorias e de forma segura, sendo compatível com a interface da maioria dos navegadores de baixa tecnologia.

No final do curso, propomos aos alunos um trabalho computacional para que possam reunir todo o conhecimento aprendido até então e aplicarem de forma mais concreta, além de terem de fazer uma prova composta por aproximadamente 5 questões que varrem todo o conteúdo abordado no curso. Além disso, após o término do período letivo, submetemos um questionário a todos os alunos (mesmo aqueles que não se formaram) com perguntas que nos permitam avaliar o curso de acordo com a opinião dos alunos. Com base nesses resultados, são realizadas as mudanças a fim de melhor atender os alunos no próximo ano. Não obstante, desde o início do curso os alunos são incentivados a manterem um contato direto com os professores, tendo total liberdade de apontarem críticas ou sugestões quando julgarem necessário.

Uma vez que o IntroComp é ministrado por aluno para alunos, os próprios alunos "instrutores" conseguem enxergar onde estão as maiores necessidades durante um curso. Com base nisso, são propostos os chamados Hacking Days (HD), que consistem em aulas extra ao curso em que ensinamos conteúdos adicionais como Scratch, Webdesign e Robótica. Como incentivo, propomos um projeto em cada HD e, para o melhor projeto, oferecemos pontuação extra, premiação, etc., de acordo com o tema abordado.

\subsection{Preparatório para a OBI}

Além do curso de Introdução à Programação, no primeiro semestre do ano seguinte ao período do curso, o projeto IntroComp também conta ainda com um preparatório para a Olímpiada Brasileira de Informática (OBI) [SBC 2015] nas modalidades de Programação (Nivel 1, 2 e Universitário), em que desde 2012 acontece sempre no primeiro semestre de cada ano. A OBI, organizada pelo Instituto de Computação da UNICAMP, é uma competição organizada nos moldes das outras olimpíadas científicas brasileiras, com medalhas como forma de premiação para os alunos mais bem colocados. A competição exige conhecimentos aprofundados em programação de computadores, com questões de níveis fáceis até mais complexos, promovendo aos alunos o desafio de resolver problemas de maneira eficiente e no menor tempo possível, fazendo uso de alguma linguagem de programação.

Com o preparatório para a OBI, todos os alunos formados no IntroComp tem a oportunidade de se dedicar ao estudo de algoritmos mais sofisticados, aprendendo de forma divertida e desafiadora conceitos importantes sobre estruturas de dados e desenvolvimento de software. Em paralelo, eles também aprendem a lidar com ambientes de pressão, tão comuns em competições de programação e, certamente, no mercado de trabalho. Diferentemente do IntroComp, o preparatório não requer uma

\footnotetext{
${ }^{3}$ http://moodle.com/
} 
prova de seleção. O curso preparatório fica destinado àqueles que se interessem pela competição ou que almejem aprender mais sobre assuntos notáveis da computação.

Durante as aulas do preparatório, que acontecem aos sábados das $9 \mathrm{~h}$ às $18 \mathrm{~h}$, são apresentadas metodologias que simulem ao máximo a prova da OBI. Como de costume em todas as aulas, no período matutino, de $9 \mathrm{~h}$ ao $12 \mathrm{~h}$, é dado aos alunos uma aula teórica sobre um assunto em específico e no período da tarde, os alunos são convidados a praticar o que foi aprendido na teoria com problemas práticos em laboratório. Além do treinamento aos sábados, os alunos também contam com os chamados "Contests", que são uma coleção de problemas de um tema específico para que os alunos possam solucionar também durante a semana.

\section{Resultados do Projeto}

O projeto tem obtido resultados cada vez mais importantes. Desde a sua criação, o aumento no número de inscrições e o aumento no número de formados são alguns desses resultados. Esses dados foram previamente apresentados no gráfico da Figura 1.

Conforme uma pesquisa realizada com os alunos que participaram do projeto (nos últimos três anos), foi possível constatar que 30\% dos alunos formados no projeto optaram por prestar vestibular para um curso na área de Informática/Computação tendo sido diretamente influenciados pelas suas respectivas participações no IntroComp. Todos eles apontaram o IntroComp como um fator determinante para essa decisão.

Com relação aos resultados dos alunos do IntroComp na OBI, podemos afirmar que estes foram expressivos. Não havia sequer participação de alunos do Espírito Santo nos quadros de méritos da competição, na modalidade de Programação, até o ano de 2011. A partir daí, com a realização do IntroComp o Estado passou a ter resultados positivos. Conforme a Tabela 1, foram obtidas diversas medalhas ao longo das edições.

Tabela 1: Premiações na OBI entre os anos de 2012 e 2014

\begin{tabular}{|c|c|c|c|c|}
\hline Ano & Nível & Ouro & Prata & Bronze \\
\hline \multirow{2}{*}{2012} & 1 & 1 & & \\
\cline { 2 - 5 } & 2 & & 1 & \\
\hline \multirow{2}{*}{2013} & 1 & & & 1 \\
\cline { 2 - 5 } & 2 & & 1 & 1 \\
\hline \multirow{2}{*}{2014} & 1 & 1 & 1 & \\
\cline { 2 - 5 } & 2 & & 1 & \\
\hline
\end{tabular}

Ainda em 2014, o projeto contou também com o destaque de um dos alunos participantes do projeto, que recebeu além do primeiro lugar geral do Brasil na OBI, a medalha de prata na Olímpiada Internacional de Informática (IoI) em Taiwan. Também foram observados resultados positivos em competições internas de programação realizadas na própria $\mathrm{UFES}^{4}$, nas quais alunos do IntroComp venceram por três anos seguidos, disputando de igual para igual com alunos da graduação. Todos esses

${ }^{4}$ http://topcom.pet.inf.ufes.br/ 
resultados podem contribuir positivamente para que o ensino de programação no Estado seja ainda mais popularizado, visto que a esses resultados são geralmente divulgados em veículos de comunicação locais, permitindo que mais alunos se sintam interessados em competir ou aprender programação.

De um modo geral, o número de inscritos e de concluintes no IntroComp cresce a cada edição, conforme o gráfico apresentado na Figura 1, à exceção de 2012 em que o projeto sofreu o impacto das greves no ensino Estadual e Federal. Mas apesar dos resultados positivos, o número de concluintes ainda é considerado pequeno. Isso devido a inúmeras razões que já foram reportadas pelos próprios alunos ao longo das edições (por meio de questionários eletrônicos). Dentre as queixas mais comuns que impossibilitam esses alunos de terminar o curso estão:

- Dificuldades no transporte até a UFES (onde as aulas são), já que, cartões de passes estudantis em transportes públicos não são válidos nos finais de semana.

- Os alunos atingidos pelo curso são alunos de instituições de ensino médio que, assim como o IntroComp, também oferecem aulas aos sábados.

- A maioria dos alunos que estão em seu último ano de ensino médio também participam de cursos preparatórios para vestibulares. Esses cursos, na maioria das vezes, são organizados com horários conflitantes com o IntroComp.

- Falta de acesso de alguns alunos ao computador fora das aulas, por razões financeiras ou outros motivos pessoais.

$\mathrm{O}$ alto índice de desistência enfrentado pelo projeto também pode estar relacionado com o processo de divulgação do mesmo. Esse processo tem sido pouco efetivo uma vez que existem na Grande Vitória em torno de 180 escolas públicas de ensino médio. Outro fator que pode estar interferindo é o fato do projeto ser executado de forma independente, sem nenhuma parceria/acompanhamento por parte das Escolas.

\section{Estendendo o Projeto}

Desde sua criação, o projeto IntroComp tem como princípio básico a ampliação do seu escopo de ação. Entendemos que quanto maior o número de alunos tendo acesso ao estudo da programação, maior será o impacto dessa iniciativa no sentido de aumentar a procura pela Computação como escolha profissional. Vale lembrar também que o desenvolvimento do pensamento computacional estimula competências que podem ser aplicadas em outras áreas do conhecimento. Adicionalmente, o domínio da programação transforma o aluno de consumidor passivo para produtor ativo de tecnologia. Independente da profissão, ele pode "dialogar" melhor com a tecnologia uma vez que ele passa a compreender melhor como ela foi desenvolvida [Hering 2014].

No entanto, desde 2013, com o aumento para duas turmas de 40 alunos, o projeto atingiu seu limite. Não somente devido a limitações de infraestrutura disponível no Departamento de Informática da UFES, mas também relacionado à sobrecarga que o projeto impõe aos alunos do PET. É importante ressaltar que esses alunos também atuam na realização de outros projetos de Ensino, Pesquisa e Extensão. Muito dessa sobrecarga deve-se à própria característica do curso que prevê uma avaliação continuada por meio de várias atividades práticas (workings) que devem ser corrigidas pelos instrutores.

Nesse contexto, em 2015, duas ações principais estão sendo realizadas no sentido de viabilizar a ampliação e o alcance do projeto, apesar das limitações descritas. 
A primeira delas consiste na integração do sistema de controle de exercícios com uma ferramenta que permite estimar de forma semi-automática as notas dos códigos submetidos. Já a segunda ação consiste no estabelecimento de parcerias para formação de "multiplicadores" do projeto. Essas duas ações são descritas a seguir.

\subsection{Avaliações semi-Automáticas dos Códigos}

Como ressaltado anteriormente, diferentes autores apontam as dificuldades do ensino e aprendizagem de programação. Para se adquirir uma aptidão em um bom nível na arte de programar, além de exercitar-se muito, é necessário que o aprendiz se aproprie de uma série de habilidades cognitivas [Pea e Kurland 1984], a começar pela complexa habilidade de compreender o problema que se pretende resolver.

Portanto, um dos grandes desafios no processo do ensino de programação é termos a capacidade de assistir prontamente cada aluno em suas necessidades individuais, sugerindo novas atividades com o objetivo de melhorar suas habilidades em programação. Em decorrência disso, estamos utilizando, ainda em fase experimental, uma ferramenta desenvolvida em [Oliveira 2013, Valentim et al. 2014]. Essa ferramenta foi integrada ao Moodle, de forma que a mesma possa analisar os códigos dos exercícios submetidos pelos alunos do IntroComp. Primeiramente, a ferramenta sugere uma amostra de códigos a serem avaliados manualmente pelo instrutor. Por meio dessa amostra estima-se as notas dos demais códigos de exercícios entregues pela turma. Com isso damos um passo adiante passando a mensurar a qualidade do código entregue como solução, quebrando o paradigma binário: gerou a solução correta - nota máxima; ou não - nota mínima.

Hoje, em nossos experimentos, já conseguimos em muitos casos poupar em até $30 \%$ o trabalho do instrutor na hora de avaliar os códigos entregues. Nosso objetivo para um futuro próximo é aumentar essa taxa e, além disso, melhorar nossa precisão na estimação de notas, que hoje tem um desvio padrão de 2 pontos em comparação às notas dadas manualmente, quando as notas variando entre 0 e 10 .

\subsection{Formação de Multiplicadores do Curso IntroComp}

Para poder ampliar o alcance do projeto, estamos buscando parcerias com outros projetos e instituições de ensino. A estratégia é que, por meio dessas parcerias, possamos formar multiplicadores do curso IntroComp, e esses multiplicadores possam replicar a metodologia do curso.

A primeira parceria estabelecida é com o Núcleo de Cidadania Digital ${ }^{5}$ (NCD) da UFES. O NCD tem como objetivo oferecer o acesso gratuito às tecnologias de informação e comunicação a moradores da região Metropolitana da Grande Vitória. Por meio dessa parceria, o NCD buscará estimular estudantes da comunidade universitária, assim como alunos do ensino médio de escolas públicas oferecendo "versões" do curso IntroComp. Para que mais pessoas possam fazer o curso, o NCD abrirá mais turmas: partir de 2016, serão abertas duas turmas adicionais por semestre (quatro turmas por ano), com aulas a serem realizadas durante a semana.

O segundo tipo de parceria que está sendo buscada é juntamente com outras Instituições de Ensino Superior do Estado. A ideia é difundir o projeto de forma que

\footnotetext{
${ }^{5}$ http://www.ncd.ufes.br/
} 
alunos de outros cursos de Computação possam reproduzir o curso para mais alunos do ensino médio. Atualmente estamos realizando um levantamento das potenciais IESs que poderiam ser parceiras do IntroComp, sobretudo fora da Grande Vitória.

\section{Trabalhos Relacionados}

A questão do ensino da programação no ensino médio já vem sendo discutida há mais de 10 anos. Pereira Júnior e Rapkiewicz (2004) representa um dos primeiros trabalhos que faz um levantamento das iniciativas nessa área. Os autores apresentam uma visão crítica em cima das pesquisas relativas ao processo de ensino-aprendizagem de programação no Brasil, concluindo que "não se pode afirmar a existência de uma solução que o resolva em sua totalidade”.

Em 2004, Pereira Junior et. al. (2005) relatam que o Grupo de Trabalho de Licenciatura em Computação (GT-3) da Sociedade Brasileira de Computação (SBC) estabeleceu como metas para 2004-2005 o desenvolvimento de experiências relativas ao ensino de programação no nível médio. Conforme ressaltado pelos autores, objetivo é desenvolver nos alunos mais precocemente competências relacionadas à programação, além de despertar neles o interesse pela área.

Em [Garcia 2008], os autores relatam uma experiência para alunos do ensino médio voltada para a resolução de problemas de lógica de programação e estrutura de dados. Essa experiência desenvolvida pela FCT-Unesp também teve como objetivo despertar o interesse dos jovens para a área de Computação e Informática, incentivando os mesmos a seguir carreira na área. Outro objetivo foi motivar os alunos a participarem da OBI. Um dos pontos observados pelos autores foi quanto à desistência dos alunos, sendo eles oriundos de escola pública. Os principais motivos foram "a necessidade de ter uma renda para ajudar a família (...) e a dificuldade em acompanhar as explicações devido a falta de raciocínio lógico, talvez pela imaturidade".

Alguns trabalhos abordam o ensino da programação pelo intermédio de estratégias pedagógicas alternativas. Em Scaico et al. (2012) os autores propõem o desenvolvimento do pensamento algorítmico por meio de uma abordagem de ensino orientado ao design. A ideia é abstrair a complexidade inerente à sintaxe das linguagens convencionais (como Phyton), e utilizar a linguagem Scratch, sendo esta mais didática para iniciantes, ainda que adequada para entender as principais estruturas para a criação de algoritmos. Já em [Marques 2011], os autores relatam a experiência de uma oficina de programação de jogos usando a linguagem Phyton. Os autores mostram que o uso de jogos foi um fator bastante motivacional.

Uma alternativa empregada é o ensino da programação por meio robótica educativa. Os trabalhos que utilizam essa estratégia se apoiam na robótica como elemento motivacional, além desta ser um instrumento de ensino integrado, permitindo aos estudantes desenvolver a capacidade de elaborar hipóteses, investigar soluções, estabelecer relações e tirar conclusões [Barros 2013][Miranda 2010].

Os trabalhos aqui listados, direta ou indiretamente, serviram de base para o desenvolvimento do projeto IntroComp. Também observamos que algumas dificuldades documentadas nos trabalhos são similares às encontradas pelo IntroComp, como as relatadas por Garcia (2008), relacionadas ao alto índice de desistência devido à falta de apoio logístico e financeiro para os alunos. 
Mas um dos principais diferenciais do projeto IntroComp em relação aos demais trabalhos relatados está no fato da atividade de ensino ser toda preparada e realizada por alunos de graduação. Com isso, é possível quebrar barreiras e inibições entre os aprendizes e instrutores, além de promover o desenvolvimento de habilidades técnicas e pedagógicas nos alunos do PET (e outros colaboradores). Certamente, isso também impõe alguns obstáculos, como a falta de experiência dos alunos instrutores. Mas com o apoio do professor Tutor responsável pelo PET e de outros professores colaboradores, é possível contornar algumas limitações técnico-pedagógicas. Além disso, em todas as aulas, tem-se um instrutor auxiliar em sala para eventual suporte.

Outro diferencial do IntroComp é a realização de Hacking Days com base nas estratégias aplicadas por outros trabalhos citados aqui, como Scratch [Scaico 2012] e robótica educacional [Barros 2013] [Miranda 2010], além do WebDesign. No caso do HD de Scratch, também adotamos uma metodologia baseada em jogos, como sugerida por Marques (2011).

\section{Considerações Finais}

Este artigo apresentou um relato de experiência descrevendo as iniciativas e estratégias do projeto IntroComp, sendo este um curso de introdução à programação preparado e realizado por alunos do PET Engenharia de Computação da UFES. A crescente participação de alunos, os resultados das pesquisas realizadas junto a ex-alunos do curso, e os resultados do Espírito Santo nos últimos anos da OBI mostram que este projeto tem grande potencial para contribuir com a iniciação de nossos alunos de ensino médio na prática da programação.

A principal dificuldade do projeto hoje é conseguir manter o aluno até a conclusão do curso. Tendo isso em vista, estamos atualmente estabelecendo uma parceria com a Sedu (Secretaria de Educação do Estado do ES). Esperamos que com a institucionalização do projeto, possamos envolver as escolas diretamente no acompanhamento dos alunos para prover maior apoio aos mesmos no decorrer do curso. Esta ação também permitirá tornar o processo de divulgação mais efetivo, podendo atingir as 175 escolas Estaduais da Grande Vitória. Outra ação será buscar formas alternativas de apoio por meio de Editais e parcerias com empresas e outras instituições, visando oferecer mais condições para que os alunos permaneçam no curso.

Com os resultados apresentados e as ações atualmente em curso, acreditamos que a ampliação do projeto seja um caminho importante para permitir que muito mais jovens possam ter acesso a esse tipo de conhecimento. Com isso, esperamos poder medir melhor o impacto dessa iniciativa na formação de nossos jovens e talvez, um dia, conseguir que o objetivo da ONG CODE.ORG [Pereira 2013], seja atingido, e que o ensino de linguagem de programação possa estar do lado de matérias tradicionais como matemática, biologia e física.

\section{Referências}

Araújo, A. (2013) "Evasão de alunos reflete na escassez de mão de obra", In: Jornal Diário do Comércio, São Paulo, Janeiro, 2013.

Barros, R. P., Fernandes, C. C., Sá, S. T., Burlamaqui, A. F., \& Gonçalves, L. M. G. (2013), "An Experience of 6 Years in Educational Robotics", In: Proceedings of the Workshop of 
Robotics on Education Proceedings (WRE 2013). Disponível em: http://www.natalnet.br/lars2013/WRE/120576.pdf

Garcia, R.E., Correia, R.C.M., Shimabukuro, M.H. (2008) "Ensino de Lógica de Programação e Estruturas de Dados para Alunos do ensino médio", In: Anais do Workshop sobre Educação em Computação (WEI'2008), Belém, PA, pp. 246-249.

Gomes, T., \& Melo, J. (2013). O Pensamento Computacional no ensino médio: Uma Abordagem Blended-Learning. In Anais do XXI Workshop sobre Educação em Computação (WEI'2013). Maceió, AL-Brasil, pp.651-660.

Hering, S. (2014) "Adolescentes veem futuro na programação", In: Olhar Digital, Acessado em 20 de abril de 2015. Disponível em http://m.olhardigital.uol.com.br/pro/noticia/adolescentesveem-futuro-na-programacao/44594.

Lockard, C.B., and Wolf, M. (2012) "Occupational employment projections to 2020." In: Monthly Lab. Rev. v.135,p. 84-108. Disponível em: http://www.bls.gov/opub/mlr/2012/01/art5full.pdf

Marques, D. L., Costa, L. F. S., de Azevedo Silva, M. A., \& Rebouças, A. D. D. S. (2011) "Atraindo Alunos do ensino médio para a Computação: Uma Experiência Prática de Introdução a Programação utilizando Jogos e Python", In: Anais do Workshop de Informática na Escola (Vol. 1, No. 1, pp. 1138-1147).

Miranda, L. C., Sampaio, F. F., \& Borges, J. A. S. (2010), "Robofacil: Especificacao e implementacao de um kit de robotica para a realidade educacional brasileira". In: Revista Brasileira de Informática na Educação, 18(3), pp. 46-58.

Oliveira, M.G. (2013), "Núcleos de Avaliações Diagnóstica e Formativa para Regulação da Aprendizagem e Programação". Tese (Doutorado) — Programa de Pós-Graduação em Engenharia Elétrica, Universidade Federal do Espírito Santo, Vitória, ES, nov.

Pea, R.D., Kurland, D. (1984) "On the Cognitive Effects of Learning Computer Programming". In: New Ideas in Psychology, v.2, n. 2, pp. 137-168. ISSN 0732-118X.

Pereira Júnior, J.C.R.P., \& Rapkiewicz, C. E. (2004), "O processo de ensino-aprendizagem de fundamentos de programação: Uma visão crítica da pesquisa no brasil". In: Anais do Workshop sobre Educação em Computação (WEI'2014), (pp. 19-21).

Pereira Júnior, J.; Rapkiewicz, C.E.; Delgado, C.; Xexeo, J.A.M. (2005) "Ensino de Algoritmos e Programação: Uma Experiência no Nível Médio". In: Anais do XIII Workshop de Educação em Computação (WEI'2005). São Leopoldo, RS, Brasil.

Pereira, L. "Escolas defendem ensino de programação a crianças e adolescentes." In: Olha Digital , 06 jun. 2013. Disponivel em: http://olhardigital.uol.com.br/noticia/escolasdefendem-ensino-de-programacao-a-criancas-e-adolescentes/35075

SBC (2015) “Olimpíada Brasileira de Informática”. http://olimpiada.ic.unicamp.br/,Abril.

Scaico, P.D. et.al. (2012)"Programação no ensino médio: Uma Abordagem de Ensino Orientado ao Design com Scratch”, In: Anais do Workshop de Informática na Escola, v.1,n.1.

Schetinger, V.C., Gottin, V.M., Pozzer, C.T. (2009) "Orientação para diminuição da evasão no curso de Ciência da Computação - Projeto Informática por Um Dia na UFSM" In: 4th Congresso Brasileiro de Extensão Universitária, Dourados, Mato Grosso do Sul.

SOFTEX (2012) "Software e Serviços de TI: A Indústria Brasileira em Perspectiva". Brasília, DF: Serifa Conhecimento e Comunicações, maio 2012.

Valentim, R. et al. (2014) "Em Busca de uma Metodologia para a Disseminação em Massa do Ensino de Programação". In: SENID - Seminário Nacional de Inclusão Digital. Passo Fundo.

Varejão, Flávio, (2014) Introdução à Programação: Uma Nova Abordagem Usando C. Elsevier Brasil. 